\title{
How much allopurinol does it take to get to target urate? Comparison of actual dose with creatinine clearance-based dose
}

\author{
Lisa K. Stamp ${ }^{1,2^{*}}$, Peter T. Chapman², Murray L. Barclay ${ }^{1,3}$, Anne Horne ${ }^{4}$, Christopher Frampton ${ }^{1}$, Paul Tan,
} Jill Drake ${ }^{1}$ and Nicola Dalbeth ${ }^{4}$

\begin{abstract}
Objective: Allopurinol dosing has frequently been limited based on creatinine clearance ( $\mathrm{CrCL}$ ), resulting in failure to achieve target serum urate (SU). The aim of this analysis was to determine how many milligrams of allopurinol above the recommended $\mathrm{CrCL}$-based dose $(\mathrm{R}+)$ are required to achieve target $\mathrm{SU}$ and to investigate the factors that influence R+.

Methods: We analysed data from participants in a 24-month open, randomized, controlled, parallel-group, comparative clinical trial. Data obtained during the 12-month dose escalation (DE) phase of the study (year 1 for DE/DE and year 2 for control/DE) were combined. R+ dose was defined as the number of milligrams of allopurinol above the CrCL-based dose at the final visit.

Results: Of the 132 participants, R+ allopurinol dose at the final visit was $\leq 100 \mathrm{mg} /$ day in 38 (28.8\%), 101-200 mg/day in 46 (34.8\%) and > $200 \mathrm{mg} /$ day in 48 participants (37.1\%). There was no significant difference between the $\mathrm{R}+$ groups in the number of participants achieving target SU. There was an increase in plasma oxypurinol and a larger percentage and absolute change in SU as R+ increased. Multivariate analysis revealed $\mathrm{CrCL}$, weight, baseline SU and allopurinol dose, were significantly positively associated with allopurinol dose at 12 months. There were no significant differences across $\mathrm{R}+$ groups in renal or liver function adverse events, although there were numerically more serious adverse events in the higher R+ groups.
\end{abstract}

Conclusion: A wide range of $\mathrm{R}+$ doses are required to achieve target SU. Four easily obtained clinical variables (baseline SU, CrCL, weight, and allopurinol dose) may be helpful to predict allopurinol dose.

Trial registration: ANZCTR, ACTRN12611000845932. Registered on 10 August 2011.

\section{Introduction}

Allopurinol remains the most commonly used urate-lowering therapy. Many clinicians have adhered to the creatinine-clearance $(\mathrm{CrCL})$ based dosing recommendation published in 1984 by Hande et al. [1] believing that this will reduce the risk of the potentially fatal allopurinol hypersensitivity syndrome (AHS). However, while higher starting doses of allopurinol have been associated with AHS [2] there is little evidence that the

\footnotetext{
* Correspondence: lisa.stamp@cdhb.health.nz; Lisa.stamp@cdhb.health.nz ${ }^{1}$ Department of Medicine, University of Otago, Christchurch, P. O. Box 4345, Christchurch 8140, New Zealand

${ }^{2}$ Department of Rheumatology, Immunology and Allergy, Christchurch Hospital, Private Bag 4710, Christchurch 8140, New Zealand

Full list of author information is available at the end of the article
}

maintenance dose, that is the dose of allopurinol required to achieve the widely recommended target urate $[3,4]$, is associated with AHS. Furthermore, a consequence of adopting a CrCL-based allopurinol dosing strategy is failure to achieve target serum urate (SU), with less than $20 \%$ of patients achieving $\mathrm{SU}<6 \mathrm{mg} / \mathrm{dL}$ on such restricted doses [5]. We have recently published results of a randomized controlled trial showing that higher than CrCL-based allopurinol doses are safe and effective in people with gout $[6,7]$.

The aims of this pre-specified secondary analysis were to determine how much above CrCL-based allopurinol dose study participants required to achieve target SU,

(c) The Author(s). 2018 Open Access This article is distributed under the terms of the Creative Commons Attribution 4.0 International License (http://creativecommons.org/licenses/by/4.0/), which permits unrestricted use, distribution, and 
factors that influence the $\mathrm{R}+$ requirement and identify any safety signal with higher $\mathrm{R}+$ requirement.

\section{Methods \\ Study design}

A 24-month open, randomized, controlled, parallelgroup, comparative clinical trial was undertaken (ACTRN12611000845932). Ethical approval was obtained from the Multi-Regional Ethics Committee, New Zealand. Written informed consent was obtained from each participant. Full methods have been reported previously [6, 7]. A total of 183 people with gout were randomized to continue current dose allopurinol for 12 months and then enter the dose escalation phase (control/DE) or to begin allopurinol dose escalation immediately $(\mathrm{DE} / \mathrm{DE})$. Allopurinol was increased monthly until SU was $<6 \mathrm{mg} / \mathrm{dl}$. For those with $\mathrm{CrCL} \leq 60 \mathrm{ml} / \mathrm{min}$, allopurinol was increased by $50 \mathrm{mg}$ increments and for those with $\mathrm{CrCL}>60 \mathrm{ml} / \mathrm{min}$ by $100 \mathrm{mg}$. For the purposes of this analysis, data obtained during the dose escalation phase of the study (year 1 for DE/DE and year 2 for control/DE participants) were combined. Thus, baseline was month 0 for DE/DE and month 12 for control/DE participants. Six participants who discontinued allopurinol due to adverse events during the study and nine participants with no post-baseline values were excluded. A further 36 participants either died or were lost to follow up before they entered or during the dose escalation phase of the study. For the remaining 132 participants the CrCL-based allopurinol dose was calculated using baseline creatinine according to the Hande criteria [1]. $\mathrm{R}+$ allopurinol dose was defined as the number of milligrams of allopurinol above baseline CrCL-based dose, and participants were grouped according to the allopurinol dose at the final visit of the dose escalation phase (i.e. month 12 for DE/DE and month 24 for control/DE) into one of the following three groups: $R+\leq 100 \mathrm{mg} /$ day, $\mathrm{R}+101-200 \mathrm{mg} /$ day, $\mathrm{R}+\geq 201 \mathrm{mg} /$ day.

\section{Adverse and serious advent event reporting}

Treatment emergent adverse events (AE) were defined as any $\mathrm{AE}$ occurring between month 0 and the end of month 12 in the DE/DE group and between month 12 and month 24 in the control/DE group. Worsening laboratory AEs were defined as those where there was an increase in $\mathrm{AE}$ grade using the Common Terminology Criteria for Adverse Events (v4.0) from the start of allopurinol dose escalation and the ensuing 12 months.

\section{Study outcomes}

The primary efficacy outcome was mean serum urate at the end of the dose escalation phase (month $12 \mathrm{DE} / \mathrm{DE}$ or month 24 control/DE). Secondary efficacy outcomes included (1) the percentage reduction in SU at the final visit of the dose escalation phase, (2) actual reduction in $\mathrm{SU}$ at the final visit of the dose escalation phase and (3) the $R+$ dose of allopurinol required to achieve $\mathrm{SU}<6 \mathrm{mg} / \mathrm{dl}$. The primary safety outcomes were serious adverse events (SAEs) and treatment emergent or worsening liver or kidney function adverse events (AEs).

\section{Statistical analysis}

Baseline demographic and clinical features were compared between $\mathrm{R}+$ groups using one-way analysis of variance, the Wilcoxon rank test, and the $\mathrm{chi}^{2}$ test as appropriate. Comparisons between those with $\mathrm{SU}<6 \mathrm{mmol} / \mathrm{l}$ and $\geq 6 \mathrm{mmol} / \mathrm{l}$ and those with or without concomitant diuretic within $\mathrm{R}+$ groups were undertaken using one-way analysis of variance. Changes in SU and plasma oxypurinol were compared between $\mathrm{R}+$ groups using one-way analysis of variance. Liver and renal function $\mathrm{AE}$ rates and SAEs were compared between $\mathrm{R}+$ groups using the chi squared test. Multiple linear regression analysis was used to test the independent associations of baseline clinical features and dose of allopurinol at 12 months. A two-tailed $p$ value $<0.05$ was taken to indicate statistical significance.

\section{Results \\ Demographics}

Baseline demographics from the 132 participants who completed the 12-month dose escalation phase of the study are outlined in Table 1. Among these 132 participants, the $\mathrm{R}+$ dose of allopurinol at the final visit was $\leq$ $100 \mathrm{mg} /$ day in 38 (28.8\%), $101-200 \mathrm{mg} /$ day in 46 (34.8\%) and $>200 \mathrm{mg} /$ day in 48 participants (37.1\%). Within these $\mathrm{R}+$ dose groups there was a wide range of $\mathrm{R}+$ doses, up to a maximum of $700 \mathrm{mg}$ in the high-dose group (Fig. 1). Those who required $\mathrm{R}+$ dose $>200 \mathrm{mg} /$ day were significantly younger, had higher baseline SU, were more likely to be obese (body mass index $\geq 30 \mathrm{~kg}$ / $\mathrm{m}^{2}$ ) and were receiving more allopurinol at the start of the dose escalation phase (Table 1).

\section{Serum urate}

There was no significant difference between the three $\mathrm{R}+$ groups in the number of participants achieving target serum urate: $\leq 100 \mathrm{mg} /$ day, 27/38 (71.1\%); $101-200 \mathrm{mg} /$ day, 38/46 (82.6\%) and $>200 \mathrm{mg} /$ day, 37/48 (77.1\%); $p=0.45$ (Table 2). There was an increase in plasma oxypurinol and a larger percentage change and absolute change in serum urate as the $\mathrm{R}+$ increased (Fig. 2a-d and Table 2). There was a larger reduction in urate in those who achieved target urate versus those who did not (Fig. 2e-g). Plasma oxypurinol was not significantly different between those at target and those not at target except at the R+250 mg/day dose (Fig. 1h). There 
Table 1 Baseline clinical features and demographic features by $\mathrm{R}+$ group as defined at month 12

\begin{tabular}{|c|c|c|c|c|}
\hline & $\mathrm{R}+\leq 100 \mathrm{mg}(n=38)$ & $\mathrm{R}+101-200 \mathrm{mg}(n=46)$ & $\mathrm{R}+>200 \mathrm{mg}(n=48)$ & $P$ value \\
\hline Age, years $^{a}$ & $61.2(13.3)$ & $64.1(9.8)$ & $55.1(11.6)$ & 0.001 \\
\hline Male, $n(\%)$ & $32(84.2)$ & $40(87.0)$ & $46(95.8)$ & 0.18 \\
\hline NZ European, n (\%) & $20(52.6)$ & $25(54.3)$ & $16(33.3)$ & 0.08 \\
\hline Duration of gout years, median (range) & $15.2(1.2-40.4)$ & $18.9(0.16-49.5)$ & $15.7(0.37-47.4)$ & 0.49 \\
\hline Baseline serum urate $\mathrm{mg} / \mathrm{dl}^{\mathrm{a}}$ & $6.6(1.6)$ & $6.6(1.0)$ & $7.6(1.5)$ & $<0.001$ \\
\hline $\mathrm{CrCl} \mathrm{ml/min}$ & $65.0(29.4)$ & $56.7(19.4)$ & $65.5(27.9)$ & 0.19 \\
\hline Weight $\mathrm{kg}^{\mathrm{a}}$, mean (SD) & $99.2(21.6)$ & $104.3(21.4)$ & $114.3(20.6)$ & 0.004 \\
\hline Body mass index, $\mathrm{kg} / \mathrm{m}^{2 a}$ & $32.2(6.3)$ & $35.2(7.9)$ & $36.6(7.3)$ & 0.23 \\
\hline Baseline allopurinol dose $\mathrm{e}^{\mathrm{a}}$ & $250(103)$ & $248(67)$ & $316(123)$ & 0.002 \\
\hline Baseline oxypurinol concentrations, $\mu \mathrm{mol} / /^{a}$ & $87.1(42.0)$ & $96.9(47.9)$ & $90.9(56.8)$ & 0.66 \\
\hline \multicolumn{5}{|l|}{ Co-existing conditions, $n(\%)$} \\
\hline Obesity $^{b}$ & $20(52.6)$ & $35(76.1)$ & $41(85.4)$ & 0.003 \\
\hline Cardiovascular disease $^{c}$ & $12(31.6)$ & $21(45.7)$ & $18(37.5)$ & 0.41 \\
\hline Diabetes mellitus & $11(28.9)$ & $16(34.8)$ & $16(33.3)$ & 0.84 \\
\hline Hypertension & $27(71.1)$ & $36(78.3)$ & $31(64.6)$ & 0.34 \\
\hline Hyperlipidemia & $25(65.8)$ & $25(54.3)$ & $25(52.1)$ & 0.41 \\
\hline Diuretic, $n(\%)$ & $12(31.6)$ & $22(47.8)$ & $18(37.5)$ & 0.30 \\
\hline
\end{tabular}

$R+$ amount of allopurinol (milligrams) above creatinine clearance-based dose, $C r C L$ creatinine clearance

${ }^{a}$ Mean (SD)

${ }^{\mathrm{b}}$ Obesity defined as body mass index $\geq 30 \mathrm{~kg} / \mathrm{m}^{2}$

${ }^{\mathrm{c} C V D}$ defined as ischemic heart disease, heart failure or peripheral vascular disease

were no significant differences in mean serum urate, or percent and absolute change in SU in those receiving and not receiving a diuretic (Additional file 1: Figure S1). However, plasma oxypurinol was significantly higher in those receiving diuretics at the larger $\mathrm{R}+$ doses. (Additional file 1: Figure S1).

\section{Adverse events}

For individual liver function tests and $\mathrm{CrCL}$, participants with $\geq 1$ adverse event during the 12-month dose escalation phase were identified. There was no significant difference across the three $\mathrm{R}+$ groups in the rates of

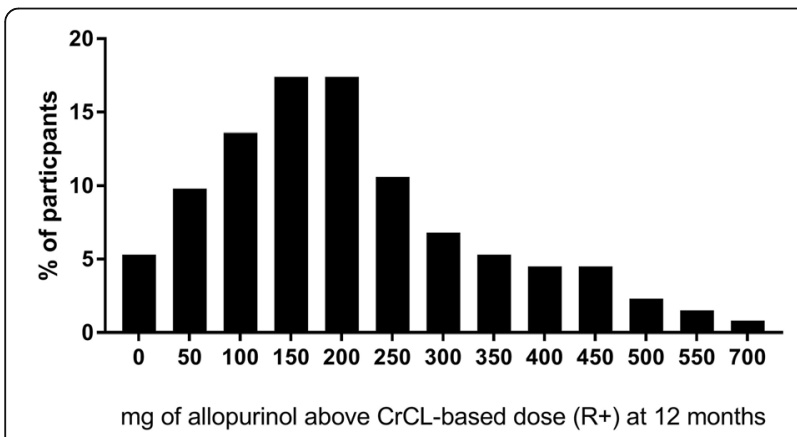

Fig. 1 Amount of allopurinol (milligrams) above the recommended creatinine clearance $(\mathrm{CrCL})$-based dose $(\mathrm{R}+)$ dose at the end of the 12-month dose escalation phase of the study abnormal alanine aminotransferase (ALT), aspartate aminotransferase (AST), gamma-glutamyl transferase (GGT), alkaline phosphatase (ALP) or changes in CrCL (Table 3 and Additional file 2: Figure S2). Serious adverse events by $\mathrm{R}+$ dose group are outlined in Table 3 . Numerically there were more participants with SAEs in the higher $\mathrm{R}+$ group than in the lower $\mathrm{R}+$ groups. However, this was not statistically significant $(p=0.44)$. None of the SAEs were thought to be allopurinol related.

There were 9 participants who died during the 12-month dose escalation phase of the study and were not included in the 132 participants used for the analysis. Details of these participants and the cause of death are outlined in Table 4. There was no significant difference in plasma oxypurinol concentration at the study visit preceding death and in the plasma oxypurinol at 12 months in the 132 participants who completed the dose escalation phase (mean (SEM) in deceased participants $176(34.6) \mu \mathrm{mol} / \mathrm{l}$ vs. $147(8.5) \mu \mathrm{mol} / \mathrm{l}$ on those who survived; $p=0.40$ ). There were no severe cutaneous adverse reactions to allopurinol.

\section{Factors predicting $\mathbf{R}+$ group at month $\mathbf{1 2}$}

We next explored a variety of clinical factors to determine which were independently associated with the $\mathrm{R}+$ group at 12 months. Multivariate analysis indicated that calculated $\mathrm{CrCL}$, baseline serum urate, weight and 
Table 2 Changes in serum urate and plasma oxypurinol between $\mathrm{R}+$ groups

\begin{tabular}{lllll}
\hline & $\mathrm{R}+\leq 100 \mathrm{mg}(n=38)$ & $\mathrm{R}+101-200 \mathrm{mg}(n=46)$ & $\mathrm{R}+>200 \mathrm{mg}(n=48)$ & $\mathrm{P}$ \\
\hline SU $<6 \mathrm{mg} / \mathrm{dl}, n(\%)$ & $27(71.1)$ & $38(82.6)$ & $37(77.1)$ & $5.5(0.17)$ \\
Mean SU, mg/dl (mean (SEM)) & $5.7(0.18)$ & $5.3(0.11)$ & $-23.6(3.1)$ & 0.19 \\
Percentage change in SU & $-2.5(4.3)$ & $-19.0(2.4)$ & $-1.95(0.26)$ & $<0.001$ \\
Delta SU, mg/dl, mean (SEM) & $-0.35(0.23)$ & $-1.4(0.20)$ & $185.1(16.9)$ & $<0.001$ \\
Plasma oxypurinol (umol/l) mean, (SEM) & $92.8(9.8)$ & $151.3(11.9)$ & $<.001$ \\
\hline
\end{tabular}

$R+$ amount of allopurinol (milligrams) above creatinine clearance-based dose, SU serum urate
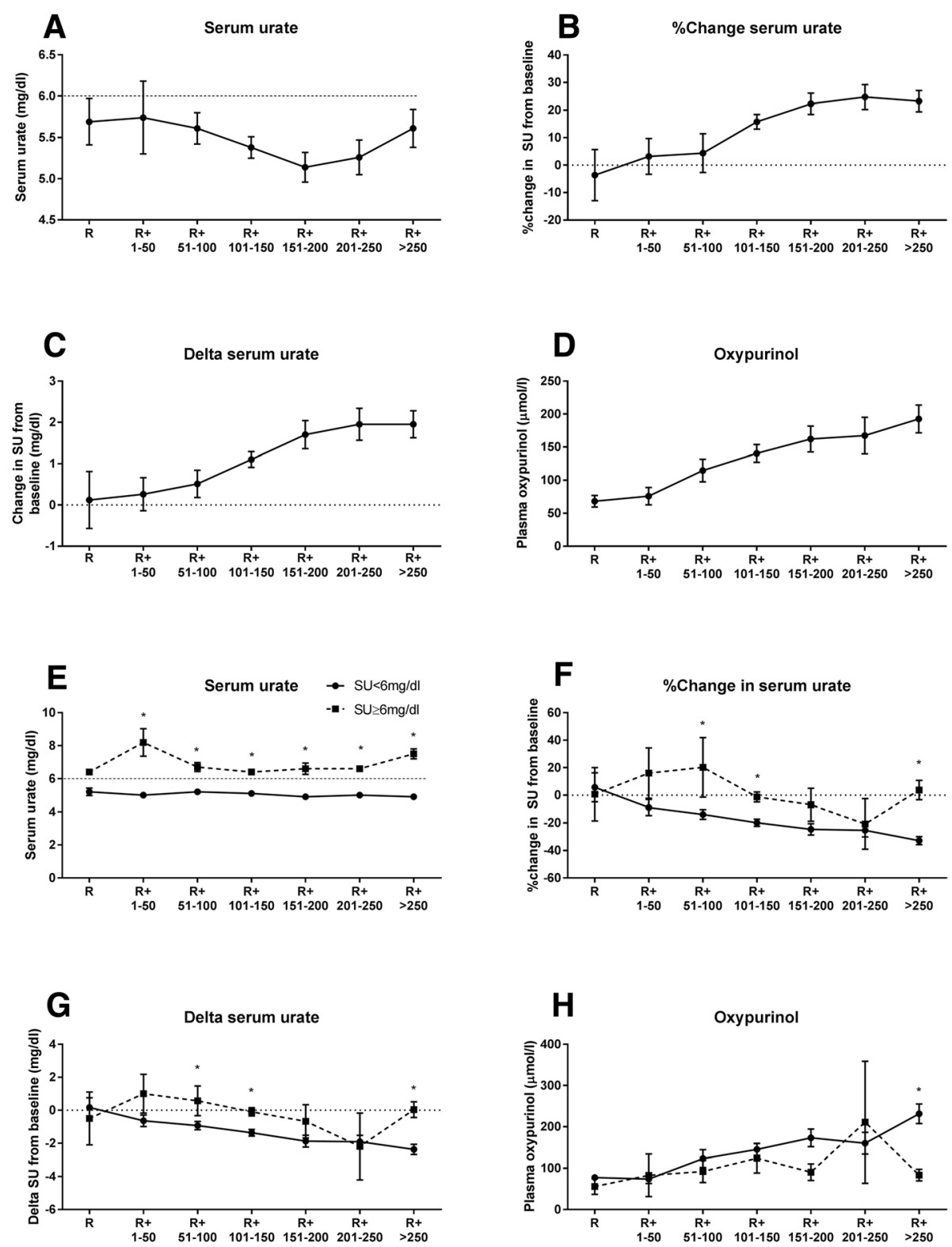

Fig. 2 Effect of increasing the amount of allopurinol (milligrams) above the recommended creatinine-clearance-based dose (R+) on serum urate (SU) (a), percentage change in SU (b), delta SU (c) and plasma oxypurinol (d) at month 12 of the dose escalation phase. Difference in SU (e), percentage change in SU (f), delta SU (g) and plasma oxypurinol (h) at month 12 of the dose escalation phase in those who did or did not achieve target SU at month 12 of the dose escalation phase. ${ }^{*} p<0.05$ 
Table 3 Abnormal renal and liver function and serious adverse events

\begin{tabular}{|c|c|c|c|c|}
\hline & $\mathrm{R}+\leq 100 \mathrm{mg}(n=38)$ & $R+101-200 \mathrm{mg}(n=46)$ & $\mathrm{R}+>200 \mathrm{mg}(n=48)$ & $P$ value \\
\hline \multicolumn{5}{|l|}{ Liver and renal function adverse events } \\
\hline GGT & $10(26.3)$ & $12(26.1)$ & $8(16.7)$ & 0.45 \\
\hline ALP & $3(7.9)$ & $1(2.2)$ & $4(8.3)$ & 0.39 \\
\hline AST & $4(10.5)$ & $3(6.5)$ & $0(0)$ & 0.09 \\
\hline ALT & $3(7.9)$ & $4(8.7)$ & $8(16.7)$ & 0.35 \\
\hline $\mathrm{CrCL}$ increase $>20 \%$ & $6(15.8)$ & $5(10.9)$ & $8(16.7)$ & 0.70 \\
\hline $\mathrm{CrCL}$ decrease $>20 \%$ & $11(28.9)$ & $9(19.6)$ & $14(29.2)$ & 0.49 \\
\hline \multicolumn{5}{|l|}{ Serious adverse events } \\
\hline & $\mathrm{R}+\leq 100 \mathrm{mg}(n=38)$ & $\mathrm{R}+101-200 \mathrm{mg}(n=46)$ & $\mathrm{R}+>200 \mathrm{mg}(n=48)$ & Total \\
\hline Cardiac disorders & 1 & $4(4)$ & $4(3)$ & 9 \\
\hline Gastrointestinal disorders & 1 & $2(2)$ & $3(3)$ & 6 \\
\hline General disorders & & 1 & $2(2)$ & 3 \\
\hline Hepatobiliary disorders & & & 1 & 1 \\
\hline Infections and infestations & & $3(3)$ & $3(3)$ & 6 \\
\hline Injury, poisoning and procedural complications & 1 & 1 & $2(2)$ & 4 \\
\hline Investigations & & 1 & & 1 \\
\hline Metabolism and nutrition & 1 & & & 1 \\
\hline Musculoskeletal & & $3(2)$ & & 3 \\
\hline Nervous system disorders & $3(2)$ & & & 3 \\
\hline Psychiatric disorders & 1 & & & 1 \\
\hline Renal and urinary disorders & & $2(2)$ & & 2 \\
\hline Respiratory & & 1 & & 1 \\
\hline Skin & & & 1 & 1 \\
\hline Total number of events (number of participants (\% of participants)) & $8(4(10.5 \%))$ & $18(8(17.4 \%))$ & $16(10(20.8 \%))$ & 42 \\
\hline
\end{tabular}

Renal and liver function data are reported as the number of participants with at least one CTCAE adverse event in the 12-month dose escalation phase of the study. Serious adverse events are reported as the number of events (number of participants)

$R+$ amount of allopurinol (milligrams) above creatinine clearance ( $\mathrm{CrCl}$ )-based dose, GGT gamma-glutamyl transferase, $A L P$ alkaline phosphatase, $A S T$ aspartate aminotransferase, $A L T$ alanine aminotransferase

baseline allopurinol dose were all significantly positively associated with allopurinol dose at 12 months $(p<0.01$ for all, $R^{2}=0.42$ ). Presence of tophi, age, diuretic use and duration of gout were not associated with $\mathrm{R}+$ group (Additional file 3: Table S1).

\section{Discussion}

This analysis has shown that the majority of people with gout who do not have target serum urate on CrCL-adjusted doses require a further increase in allopurinol dose of $200 \mathrm{mg}$ or less to achieve target serum

Table 4 Details of the nine participants who died during the dose escalation phase of the study

\begin{tabular}{|c|c|c|c|c|c|c|c|}
\hline $\begin{array}{l}\text { Month } \\
\text { of death }\end{array}$ & $\begin{array}{l}\text { Baseline } \\
\text { allopurinol dose }\end{array}$ & $\begin{array}{l}\text { Allopurinol } \\
\text { dose at death }\end{array}$ & $\begin{array}{l}\mathrm{R}+ \\
\text { (mg/day) }\end{array}$ & $\begin{array}{l}\text { Baseline } \mathrm{CrCL} \\
(\mathrm{ml} / \mathrm{min})\end{array}$ & $\begin{array}{l}\text { Urate }(\mathrm{mmol} / \mathrm{l}) \text { at } \\
\text { visit preceding death }\end{array}$ & $\begin{array}{l}\text { Oxypurinol }(\mu \mathrm{mol} / \mathrm{l}) \\
\text { at visit preceding death }\end{array}$ & Cause of death \\
\hline 11 & 200 & 400 & +300 & 28 & 0.34 & 341 & Heart failure \\
\hline 11 & 300 & 400 & +250 & 32 & 0.37 & 64 & Lung infection \\
\hline 3 & 200 & 250 & +100 & 37 & 0.45 & 205 & Heart failure \\
\hline 9 & 600 & 400 & +150 & 84 & 0.64 & 0 & Acute coronary syndrome \\
\hline 5 & 150 & 250 & +50 & 65 & 0.37 & 288 & Heart failure \\
\hline 10 & 300 & 300 & +150 & 46 & 0.40 & 201 & Cardiac arrest \\
\hline 9 & 200 & 250 & +150 & 31 & 0.4 & 164 & Heart failure \\
\hline 4 & 600 & 600 & +400 & 56 & 0.46 & 179 & Aortic dissection \\
\hline 2 & 300 & 300 & +100 & 51 & 0.40 & 139 & Heart failure \\
\hline
\end{tabular}

$R+$ amount of allopurinol (milligrams) above creatinine clearance-based dose 
urate. The multivariate analysis has identified four readily available clinical variables that are associated with requiring higher allopurinol doses; SU, CrCL, weight and allopurinol dose.

It has previously been reported that there is a ceiling effect with allopurinol, such that no matter how high the allopurinol dose there is a limit to the urate-lowering effect [8]. Consistent with this concept, our analysis has shown a flattening of the SU decrease, percentage change in SU and absolute change in serum urate once the $\mathrm{R}+$ dose is $>200 \mathrm{mg}$ daily (Fig. 2). However, this may in part be due to poor adherence in the higher $\mathrm{R}+$ groups, noting the lower plasma oxypurinol concentrations in those failing to achieve target urate. The number of tablets required for some of the higher $\mathrm{R}+$ doses may be a barrier to adherence.

While there was no increase in AEs associated with higher $\mathrm{R}+$ doses, there were numerically more SAEs. These SAEs were not deemed allopurinol-related. In addition, the $\mathrm{R}+$ doses of the nine individuals who died during the dose escalation phase of the study ranged across all $\mathrm{R}+$ groups, and plasma oxypurinol concentrations were not significantly higher in these patients than in those who completed the study. The large numbers and types of SAEs are not surprising considering the high prevalence of co-morbidities in the study population. As anticipated, there were no cases of AHS despite the $\mathrm{R}+$ doses; this is not unexpected as participants had been tolerating allopurinol for at least one month prior to study entry and the study was not powered to detect this rare event.

Of particular importance were the cardiovascular SAEs. The role of the xanthine oxidase inhibitors allopurinol and febuxostat in cardiovascular events has been the subject of much interest. The recently published study, the Cardiovascular Safety of Febuxostat and Allopurinol in Patients with Gout and Cardiovascular Morbidities, reported no significant difference between allopurinol and febuxostat in the primary composite endpoint of cardiovascular events in people with gout and established cardiovascular comorbidities at baseline. However, there was significantly increased risk of cardiac and all-cause mortality with febuxostat compared to allopurinol [9]. Of importance in this study of 6190 people, the starting dose of febuxostat was $40 \mathrm{mg}$ daily with an increase to $80 \mathrm{mg} /$ daily if SU was $>6 \mathrm{mg} / \mathrm{dl}$ at week 2 , the starting dose of allopurinol was dependent on kidney function and allopurinol was systematically increased to achieve target urate $<6 \mathrm{mg} / \mathrm{dl}$ with a maximum dose of $600 \mathrm{mg}$ daily, reduced to $400 \mathrm{mg}$ daily in those with estimated creatinine clearance of 30 to $<60 \mathrm{ml} / \mathrm{min}$ [9]. Whether there is a protective effect of allopurinol remains unclear. A recent meta-analysis of 81 randomized controlled trials reported that allopurinol was associated with a lower risk of myocardial infarction (OR 0.38, 95\% CI 0.17-0.83), hypertension (OR 0.32, 95\% CI $0.18-0.58$ ), total cardiovascular events (OR 0.48, 95\% CI $0.31-0.75)$ and serious total cardiovascular events (OR $0.56,95 \%$ CI $0.36-0.86, I^{2}=44 \%$ ) compared to placebo or no treatment. In comparison, febuxostat and topiroxostat were not associated with an increased or decreased risk of cardiovascular events [10]. Interestingly, on meta-regression higher doses of allopurinol (>300 mg/ day) were associated with higher risk of total cardiovascular events [10]. In the current study 8/9 deaths were cardiovascular-related. Further studies are required to determine whether allopurinol has a protective effect against cardiovascular events and whether this is dose-related.

There are some limitations to this study. The populations in this study had a high prevalence of co-morbidities which is fairly typical for people with gout. One would anticipate that in a heathier population with gout, higher $\mathrm{R}+$ does would possibly be better tolerated. In addition, this was a pre-planned secondary analysis of the main study.

\section{Conclusion}

A wide range of $\mathrm{R}+$ doses are required to achieve target SU. Four easily obtained clinical variables may be helpful in predicting higher allopurinol dose requirement. The relationship between higher $\mathrm{R}+$ dose and SAEs is unclear.

\section{Additional files}

Additional file 1: Figure S1. Effect of diuretic on (A) serum urate, (B) \% change in serum urate, $(C)$ delta serum urate and (D) plasma oxypurinol by $R+$ group at month 12 of the dose escalation phase. ${ }^{*} p<0.05$. (JPG $560 \mathrm{~kb}$ )

Additional file 2: Figure S2. Creatinine clearance $(\mathrm{CrCL})$ at 12 months by $\mathrm{R}+$ group. (TIF $168 \mathrm{~kb}$ )

Additional file 3: Table S1. Multivariate analysis of factors associated with $\mathrm{R}+$ group at month 12 . (DOCX $14 \mathrm{~kb}$ )

\section{Abbreviations}

AE: Adverse event; AHS: Allopurinol hypersensitivity syndrome; ALP: Alkaline phosphatase; ALT: Alanine aminotransferase; AST: Aspartate aminotransferase; C: Control; Cl: Confidence interval; CrCL: Creatinine clearance; D: Daily;

DE: Dose escalation; GGT: Gamma-glutamyl transferase; mg: Milligrams; ml/ min: Millilitres per minute; OR: Odds ratio; R+: Milligrams of allopurinol above creatinine clearance-based dose; SAE: Serious adverse event; SEM: Standard error of the mean; SU: Serum urate

\section{Acknowledgements}

We are grateful to the Health Research Council of New Zealand Independent Data Safety Monitoring Committee for monitoring the study.

\section{Funding}

This study was funded by the Health Research Council of New Zealand. The funding body had no role in the design, and collection, analysis, interpretation of data or in writing the manuscript.

Availability of data and materials

The datasets used and/or analysed during the current study are available from the corresponding author on reasonable request. 


\section{Authors' contributions}

LS: literature search, study design, data collection, data analysis, data interpretation, manuscript preparation. PC: study design, data collection, data analysis, data interpretation, manuscript preparation. MB: study design, data analysis, data interpretation, manuscript preparation. $\mathrm{AH}$ : data collection, data analysis, data interpretation, manuscript preparation. CF: study design, data analysis, data interpretation, manuscript preparation. PT: data collection, data analysis, data interpretation, manuscript preparation. JD: study design, data collection, data analysis, data interpretation, manuscript preparation. ND: literature search, study design, data collection, data analysis, data interpretation, manuscript preparation. All authors read and approved the final manuscript.

\section{Ethics approval and consent to participate}

Ethical approval was obtained from the Multi-Regional Ethics Committee, New Zealand. Written informed consent was obtained from each participant.

\section{Consent for publication}

Not applicable.

\section{Competing interests}

Prof. Stamp reports grants from the Health Research Council of New Zealand during the conduct of the study; grants from Ardea Biosciences, grants from the Health Research Council of New Zealand, outside the submitted work; Dr Chapman has nothing to disclose: Prof. Barclay has nothing to disclose; Dr Horne reports grants from the Health Research Council of New Zealand during the conduct of the study; Prof. Frampton has nothing to disclose; Mrs Drake has nothing to disclose; Mr Tan has nothing to disclose; Prof. Dalbeth reports grants from the Health Research Council of New Zealand during the conduct of the study; grants from the Health Research Council of New Zealand, grants and personal fees from AstraZeneca, grants and personal fees from Ardea Biosciences, grants from Amgen, personal fees from Takeda, personal fees from Horizon and personal fees from Kowa, outside the submitted work.

\section{Publisher's Note}

Springer Nature remains neutral with regard to jurisdictional claims in published maps and institutional affiliations.

\section{Author details}

${ }^{1}$ Department of Medicine, University of Otago, Christchurch, P. O. Box 4345 , Christchurch 8140, New Zealand. 'Department of Rheumatology, Immunology and Allergy, Christchurch Hospital, Private Bag 4710, Christchurch 8140, New Zealand. ${ }^{3}$ Department of Clinical Pharmacology, Christchurch Hospital, Private Bag 4710, Christchurch 8140, New Zealand. ${ }^{4}$ Department of Medicine, University of Auckland, Private Bag 92019, Auckland, New Zealand.

Received: 19 July 2018 Accepted: 29 October 2018

Published online: 16 November 2018

\section{References}

1. Hande K, Noone R, Stone W. Severe allopurinol toxicity. Description and guidelines for prevention in patients with renal insufficiency. Am J Med. 1984;76:47-56.

2. Stamp L, Taylor W, Jones P, Dockerty J, Drake J, Frampton C, Dalbeth N. Starting dose, but not maximum maintenance dose, is a risk factor for allopurinol hypersensitivity syndrome: a proposed safe starting dose of allopurinol. Arthritis Rheum. 2012;64(8):2529-36.

3. Richette $P$, Doherty M, Pascual E, Barskova V, Becce F, Castaneda-Sanabria J, Coyfish M, Guillo S, Janesen T, Janssens H, et al. 2016 Updated EULAR evidence-based recommendations for the management of gout. Ann Rheum Dis. 2017;76(1):29-42.

4. Khanna D, Fitzgerald J, Khanna P, Sangmee B, Singh M, Neogi T, Pillinger M, Merill J, Lee S, Prakash S, et al. 2012 American College of Rheumatology guidelines for the management of gout. Part 1: systematic nonpharmacologic and pharmacologic therapeutic approaches to hyperuricaemia. Arthritis Care Res. 2012;64(10):1431-46.

5. Dalbeth N, Kumar S, Stamp LK, Gow P. Dose adjustment of allopurinol according to creatinine clearance does not provide adequate control of hyperuricaemia in patients with gout. J Rheumatol. 2006;33(8):1646-50.

6. Stamp L, Chapman P, Barclay M, Horne A, Frampton C, Tan P, Drake J, Dalbeth N. A randomised controlled trial of the efficacy and safety of allopurinol dose escalation to achieve target serum urate in people with gout. Ann Rheum Dis. 2017;76:1522-8.

7. Stamp L, Chapman P, Barclay M, Horne A, Frampton C, Tan P, Drake J, Dalbeth N. Allopurinol dose escalation to achieve serum urate below $6 \mathrm{mg} /$ dl: an open label extension study. Ann Rheum Dis. 2017;76:2065-70.

8. Day R, Graham G, Hicks M, McLachlan A, Stocker S, Williams K. Clinical pharmacokinetics and pharmacodynamics of allopurinol and oxypurinol. Clin Pharmacokinet. 2007:46(8):623-44.

9. White W, Saag K, Becker M, Borer J, Gorelick P, Whelton A, Hunt B, Castillo M, Gunawardhana L. Cardiovascular safety of febuxostat or allopurinol in patients with gout. N Engl J Med. 2018. https://doi.org/10.1056/NEJMoa1710895.

10. Bredemeier $M$, Lopes $L$, Eisenreich $M$, Hickmann $S$, Bongiorno $G$, d'Avila R. Xanthine oxidase inhibitors for prevention of cardiovascular events: a systematic review and meta-analysis of randomized controlled trials. BMC Cardiovasc Disord. 2018;18:24.

\section{Ready to submit your research? Choose BMC and benefit from:}

- fast, convenient online submission

- thorough peer review by experienced researchers in your field

- rapid publication on acceptance

- support for research data, including large and complex data types

- gold Open Access which fosters wider collaboration and increased citations

- maximum visibility for your research: over $100 \mathrm{M}$ website views per year

At BMC, research is always in progress.

Learn more biomedcentral.com/submissions 